\title{
SAÚDE DO TRABALHADOR DE ENFERMAGEM: RESGATE DA PRODUÇÃO CIENTÍFICA
}

\author{
Vinicius Gomes Barros ${ }^{1}$, Cristiane Helena Gallasch², Kátia Pontes Remijo ${ }^{3}$, Karyme Lucila Jabra Lima \\ Patricia Campos Pavan Baptista ${ }^{5}$, Vanda Elisa Andres Felli ${ }^{6}$
}

\begin{abstract}
RESUMO: Este estudo teve como objetivo resgatar e analisar a produção de conhecimento publicada sobre a saúde do trabalhador de enfermagem e de saúde gerada pelos integrantes do Grupo de Estudos Sobre a Saúde do Trabalhador de Enfermagem e Saúde. Trata-se de um estudo documental, descritivo e exploratório, com análise quantitativa dos dados referentes à produção entre 1997 e 2016. Em 20 anos, foram geradas 343 publicações. A análise mostrou o predomínio de publicações de pesquisadores doutores (146; 42,57\%), seguidos dos mestres $(105 ; 30,61 \%)$ e graduandos $(62 ; 18,08 \%)$, sendo a maioria "resumos e resumos expandidos publicados em anais" (172; 50,15\%), seguidos de artigos publicados em periódicos científicos $(74 ; 21,57 \%)$. A temática "Exposições a cargas de trabalho e/ou Processo de desgaste no Trabalho" foi a predominante, evidenciando os riscos à saúde do trabalhador. Conclui-se que o grupo de pesquisa apresentou produção científica expressiva no período estudado, contribuindo com o avanço do conhecimento científico sobre o tema em foco.
\end{abstract}

DESCRITORES: Enfermagem; Grupos de pesquisa; Saúde do trabalhador; Pesquisa; Pessoal de saúde; Desenvolvimento de pessoal.

\section{NURSING WORKER HEALTH: IDENTIFICATION OF SCIENTIFIC PRODUCTION}

\begin{abstract}
The present study aimed to identify and analyze the scientific publications on health and nursing worker health generated by members of the Study Group on Occupational Health of Nursing and Health Personnel (GESTES). Descriptive-exploratory, documentary study of quantitative approach of data related to scientific publications of 1997 and 2016 . In 20 years, 343 publications were generated. Analysis showed a predominance of publications by doctors $(146 ; 42,57 \%)$, followed by masters (105; 30, 61\%) and graduate students $(62 ; 18,08 \%)$, most of them "abstracts and extended abstracts published in annals" (172; 50.15\%), followed by articles published in scientific journals $(74 ; 21,57 \%)$. The theme "Exposure to workloads and/or work wear Process" prevailed, demonstrating the risks to worker health. It is concluded that the research group identified a significant volume of scientific publications on the referred theme during the study period.
\end{abstract}

DESCRIPTORS: Nursing; Research groups; Worker health; Research; Health personnel; Personnel development.

\section{SALUD DEL TRABAJADOR DE ENFERMERÍA: RESCATE DE LA PRODUCCIÓN CIENTÍFICA}

RESUMEN: Estudio cuyo objetivo fue rescatar y analizar la producción de conocimiento publicada acerca del tema salud del trabajador de enfermería y salud generada por los integrantes del Grupo de Estudios Sobre Salud del Trabajador de Enfermería y Salud. Es un estudio documental, descriptivo y exploratorio, con análisis cuantitativo de los datos referentes a la producción entre 1997 y 2016. En 20 años, fueron producidas 343 publicaciones. El análisis ha apuntado predominio de publicaciones de doctores (146; $42,57 \%)$, maestros $(105 ; 30,61 \%)$ y graduandos $(62 ; 18,08 \%)$, siendo la mayoría "resúmenes y resúmenes expandidos publicados en anales" (172; 50,15\%), además de artículos publicados en periódicos científicos $(74 ; 21,57 \%)$. La temática "Exposiciones a cargas de trabajo y/o Proceso de desgaste en el Trabajo" fue la predominante, evidenciándose los riesgos a la salud del trabajador. Se concluye que el grupo de investigación ha presentado producción científica expresiva en el dicho periodo, contribuyendo con el desarrollo del conocimiento científico acerca del tema en cuestión.

DESCRIPTORES: Enfermería; Grupos de investigación; Salud del trabajador; Investigación; Equipo de salud; Desarrollo de personal.

${ }^{1}$ Enfermeiro. Doutorando em Gerenciamento em Enfermagem. Universidade de São Paulo. São Paulo, SP, Brasil.

${ }^{2}$ Enfermeira. Pós-doutora em Enfermagem. Docente de Enfermagem da Universidade do Estado do Rio de Janeiro, Rio de Janeiro, RJ, Brasil.

${ }^{3}$ Enfermeira. Mestranda em Gerenciamento em Enfermagem. Universidade de São Paulo. São Paulo, SP, Brasil.

${ }^{4}$ Enfermeira. Mestre em Enfermagem. Docente de Enfermagem do Centro Universitário de Várzea Grande. Várzea Grande, MT, Brasil.

${ }^{5}$ Enfermeira. Pós-doutora em Enfermagem. Livre-Docente. Docente de Enfermagem da Universidade de São Paulo. São Paulo, SP, Brasil.

${ }^{6}$ Enfermeira. Doutora em Enfermagem. Livre-Docente. Docente de Enfermagem da Universidade de São Paulo. São Paulo, SP, Brasil.

Autor Correspondente:

Recebido: 28/11/2016

Vinicius Gomes Barros

Finalizado: 30/06/2017

Universidade de São Paulo

Av. Dr. Enéas de Carvalho Aguiar, 419 - 05403-000 - São Paulo, SP, Brasil

E-mail: viniciusvgb@usp.br 


\section{- INTRODUÇÃO}

O desenvolvimento de pesquisas em Enfermagem constitui importante estratégia para seu fortalecimento na ciência e profissão, com uma prática profissional sustentada por uma contínua busca de novos conhecimentos ${ }^{(1)}$. A produção científica em enfermagem no Brasil, assim como o número de periódicos científicos relacionados à área, intensificou-se a partir da década de 70, fruto da Reforma Universitária de $1968^{(2)}$.

Durante a década de1980, estudos relacionados à Saúde do Trabalhador de Enfermagem apresentaram um aumento significativo, como resultado de uma série de mudanças que ocorreram no macro e microssistemas sociais envolvendo duas grandes áreas: saúde e educação. Esse aumento decorreu também de uma maior aproximação das práticas e saberes relacionados à saúde do trabalhador de enfermagem, tendo contribuído para este fato a estruturação e expansão dos cursos de mestrado e doutorado no país ${ }^{(3)}$.

Ainda nesta área, a pesquisa de enfermagem vem ganhando destaque internacionalmente, avançando na compreensão do trabalho destes profissionais na prevenção de agravos, proporcionando acesso a cuidados de qualidade, e gerenciando ambientes e o retorno ao trabalho(4).

A pesquisa vai além dos resultados propriamente ditos, constituindo um instrumento que proporciona aos acadêmicos a possibilidade de tornarem-se profissionais autônomos, críticos e criativos. O desenvolvimento de trabalhos científicos, desde os primeiros anos de formação, pode aproximar os alunos dos problemas reais da sociedade, aos quais apliquem os conhecimentos e habilidades adquiridas, de modo a formar uma atitude científica extensível à atividade profissional ${ }^{(5)}$.

A necessidade de envolver enfermeiros nas atividades de pesquisa e extensão é reconhecida, além de estimular os alunos a integrar os grupos de pesquisa com um objetivo comum, de atender às demandas da sociedade. Além disso, a divulgação dos resultados das pesquisas consiste em uma das etapas do processo da produção do conhecimento; outras estratégias devem também ser implementadas para a formação de recursos humanos e melhoria da assistência de enfermagem ${ }^{(6-7)}$.

O Grupo de Estudos Sobre a Saúde do Trabalhador de Enfermagem e Saúde (GESTES), vinculado ao Departamento de Orientação Profissional (ENO) da Escola de Enfermagem da Universidade de São Paulo (EEUSP), certificado pelo Diretório de Grupos de Pesquisas do Conselho Nacional de Desenvolvimento Científico e Tecnológico (CNPq), iniciou suas atividades científicas em 1997 e vem produzindo conhecimento sobre este tema, por meio de atividades direcionadas por seus líderes e de alunos de diferentes níveis, ou seja, graduação e pós-graduação (lato e estrito senso). Ainda, suas líderes integram a Rede de Pesquisa de Gerenciamento de Enfermagem (REPEGENF).

Os encontros do Grupo acontecem com interação entre líderes, pesquisadores e alunos, em reuniões mensais, em que, obedecendo todos os preceitos éticos, são desenvolvidas pesquisas de interesse do grupo, métodos de abordagem, participação em eventos, publicações - uma série de atividades que promovem o desenvolvimento de investigações e a formação de recursos humanos para a pesquisa e a pós-graduação. As reuniões do Grupo acontecem no Departamento de Orientação Profissional da EEUSP, loccus de trabalho das líderes.

Os projetos de pesquisa desenvolvidos no Grupo, além de sua produção científica, têm incrementado os intercâmbios com outros grupos de pesquisa, nacionais e internacionais, com desenvolvimento de projetos locais e multicêntricos. Um exemplo é o projeto multicêntrico "Sistema de Monitoramento da Saúde do Trabalhador de Enfermagem" (SIMOSTE), desenvolvido pelos pesquisadores do grupo, realizando estudos que abordam a saúde dos trabalhadores de enfermagem e de saúde, com o objetivo de monitorá-los com vistas à vigilância à saúde ${ }^{(8)}$.

Após 20 anos de existência e trabalho desenvolvido pelos seus membros, o GESTES apresenta produção científica significativa e em fase de consolidação que, no entanto, ainda não está suficientemente incorporada pelas diferentes realidades de trabalho de enfermagem. Portanto, nos motivamos a resgatar essa produção para dar maior visibilidade, assim como detectar lacunas de conhecimento que possam direcionar outros estudos.

Assim, o objetivo desse estudo foi resgatar e analisar a produção de conhecimento publicada sobre 
a saúde do trabalhador de enfermagem e de saúde gerada pelos integrantes do Grupo de Estudos Sobre a Saúde do Trabalhador de Enfermagem e Saúde.

\section{MÉTODO}

Trata-se de um estudo documental, descritivo e exploratório, com análise quantitativa dos dados referentes à produção do Grupo de Estudos Sobre Saúde do Trabalhador de Enfermagem e Saúde de 1997 a 2016. É definido como estudo descritivo e exploratório, uma vez que tem a característica de observar, classificar e descrever o fenômeno ${ }^{(9)}$.

Para alcançar o objetivo proposto, este estudo foi desenvolvido em três etapas. A primeira consistiu-se da identificação de toda a produção científica publicada, conduzida sob a orientação dos professores líderes, entre 1997 e 2016, por meio da listagem dos pesquisadores envolvidos no grupo, realizada pelos líderes, consulta aos currículos dos membros atuais e egressos do Grupo, também disponíveis na Plataforma Lattes do CNPq; busca nas plataformas DEDALUS da Universidade de São Paulo e no Research Gate. Nessa etapa, foram identificadas 343 produções científicas publicadas, que constituíram a população do estudo. Constituiu critérios de inclusão, toda a produção publicada dos membros do grupo, durante o período de coleta. Os critérios de exclusão foram publicações que não disponibilizaram acesso ao resumo ou ao texto na íntegra, e publicações de membros do grupo com temática diferente da estabelecida como eixo norteador.

A segunda etapa foi composta pela construção de um banco de dados, com apoio do software Microsoft Excel ${ }^{\circledR}$. Esse banco permitiu verificar duplicidade de produções, uma vez que poderia ser captada em diferentes fontes de dados. As informações contidas no banco referiram-se a: nome do pesquisador; formação; título da produção, tipo de produção, autores, vertente teórico-metodológica, ano e referência.

A terceira etapa consistiu na análise dos tipos de publicações e temáticas abordadas durantes o período, nas publicações científicas relacionadas, com análise quantitativa dos dados encontrados e qualitativa dos temas.

\section{- RESULTADOS}

Nos 20 anos de existência, o GESTES contou com 61 integrantes, entre alunos e pesquisadores. Estes geraram 343 publicações, resultantes de estudos na área da saúde do trabalhador em enfermagem e de saúde, distribuídas entre diversos níveis de formação/titulação, conforme apresentado na Figura 1.

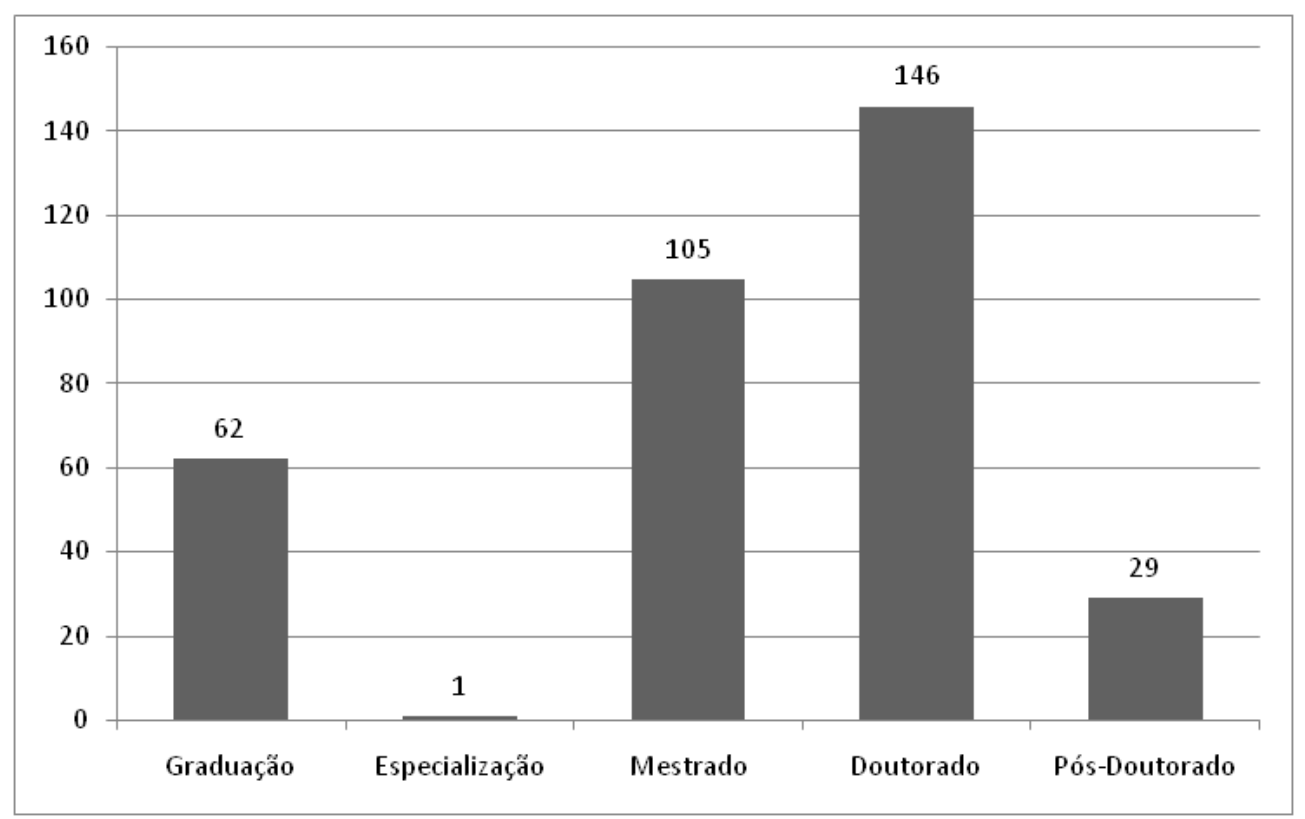

Figura 1 - Distribuição das publicações por formação/titulação dos autores do GESTES. São Paulo, SP, Brasil, 2017 
Observa-se que houve predomínio de publicações de pesquisadores doutores $(146 ; 42,57 \%)$, seguidos por mestres $(105 ; 30,61 \%)$ e graduandos $(62 ; 18,08 \%)$.

Devido à heterogênea composição do grupo, que conta com alunos e pesquisadores com diferentes titulações, há uma diversidade de tipos de publicações produzidas pelos membros do grupo no período estudado, de acordo com a Figura 2.

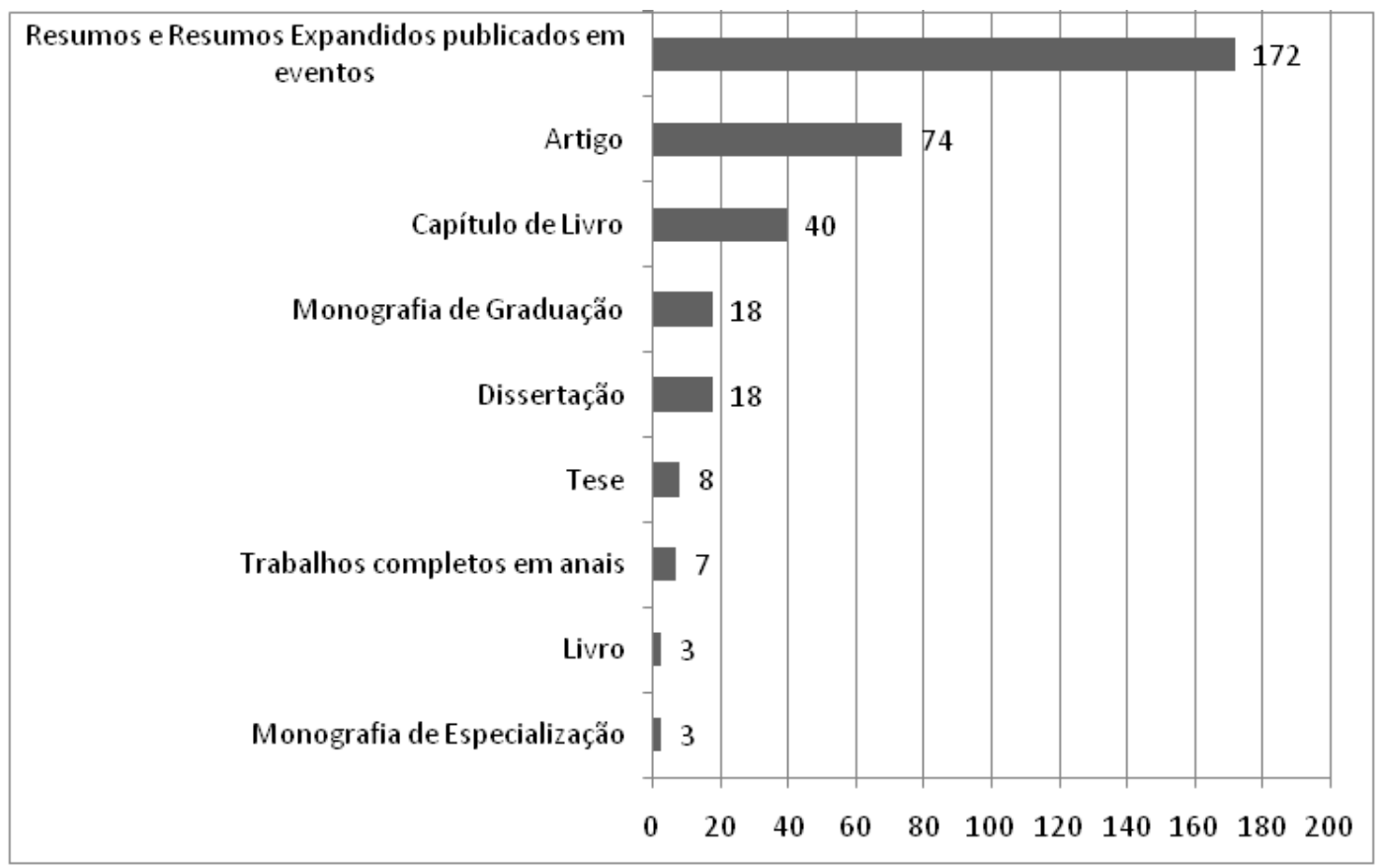

Figura 2 - Distribuição dos tipos de publicações do GESTES, entre 1997 e 2016. São Paulo, SP, Brasil, 2017

Foi verificada a prevalência de "resumos e resumos expandidos publicados em anais", com total de 172 trabalhos publicados $(50,15 \%)$, e de artigos publicados em periódicos científicos, com 74 manuscritos $(21,57 \%)$.

Assim como observado na literatura apresentada anteriormente, a produção do grupo vem crescendo ao longo dos anos estudados, como demonstrado na Figura 3.

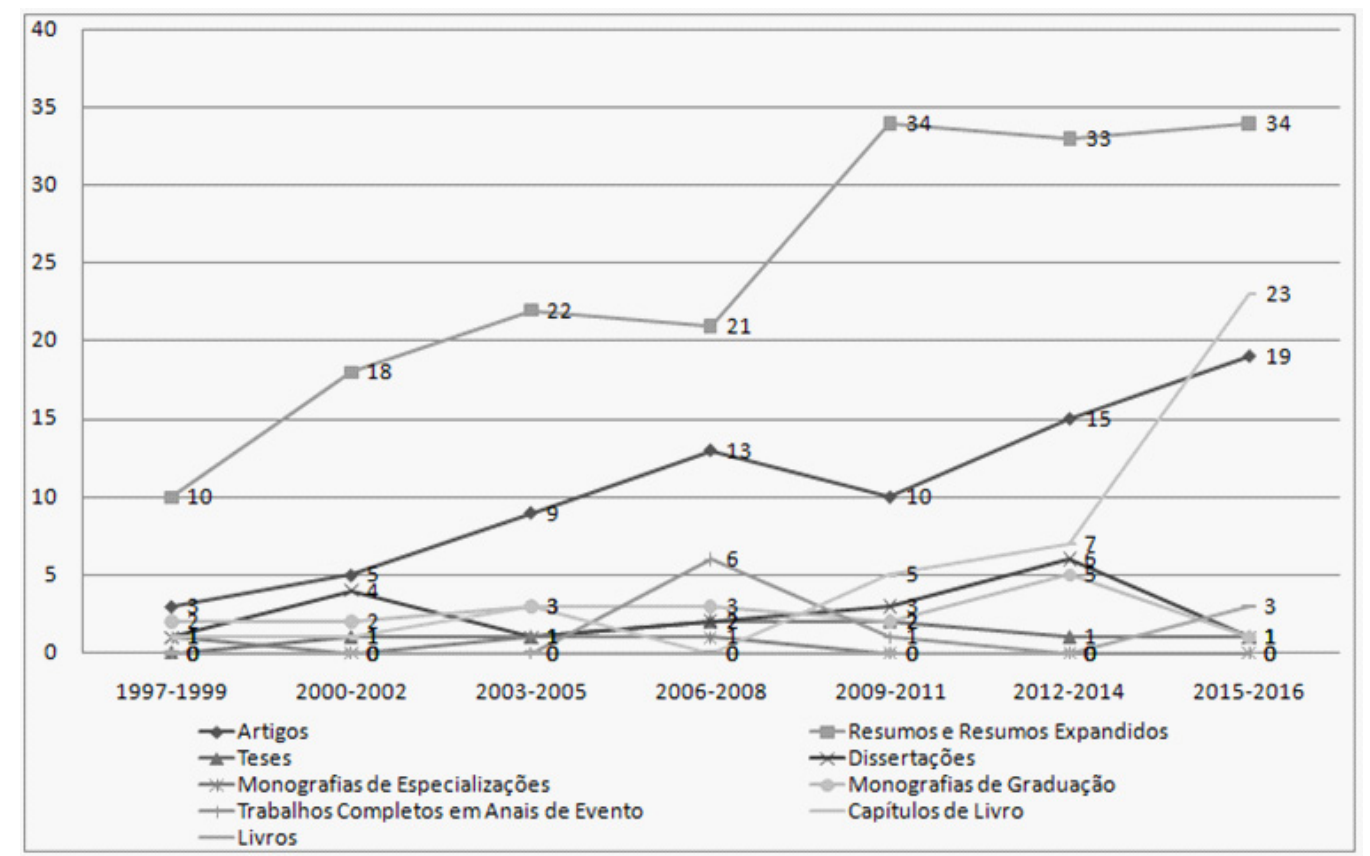

Figura 3 - Tipos de publicações científicas resultantes do GESTES entre 1997 e 2016. São Paulo, SP, Brasil, 2017 
A maior parte das publicações apresentam crescimento expressivo, os Resumos e Resumos Expandidos apresentam um crescimento de 10 publicações entre os anos de 1997-1999 para 34 entre os anos de 2015-2016. Neste mesmo período, houve o crescimento de 03 para 19 artigos científicos publicados e de 01 para 23 capítulos de livro publicados. As outras publicações, embora apresentem oscilações mais constantes, apresentaram leve crescimento em sua produção bibliográfica.

As temáticas das publicações do grupo no período foram descritas e são apresentadas na Figura 4.

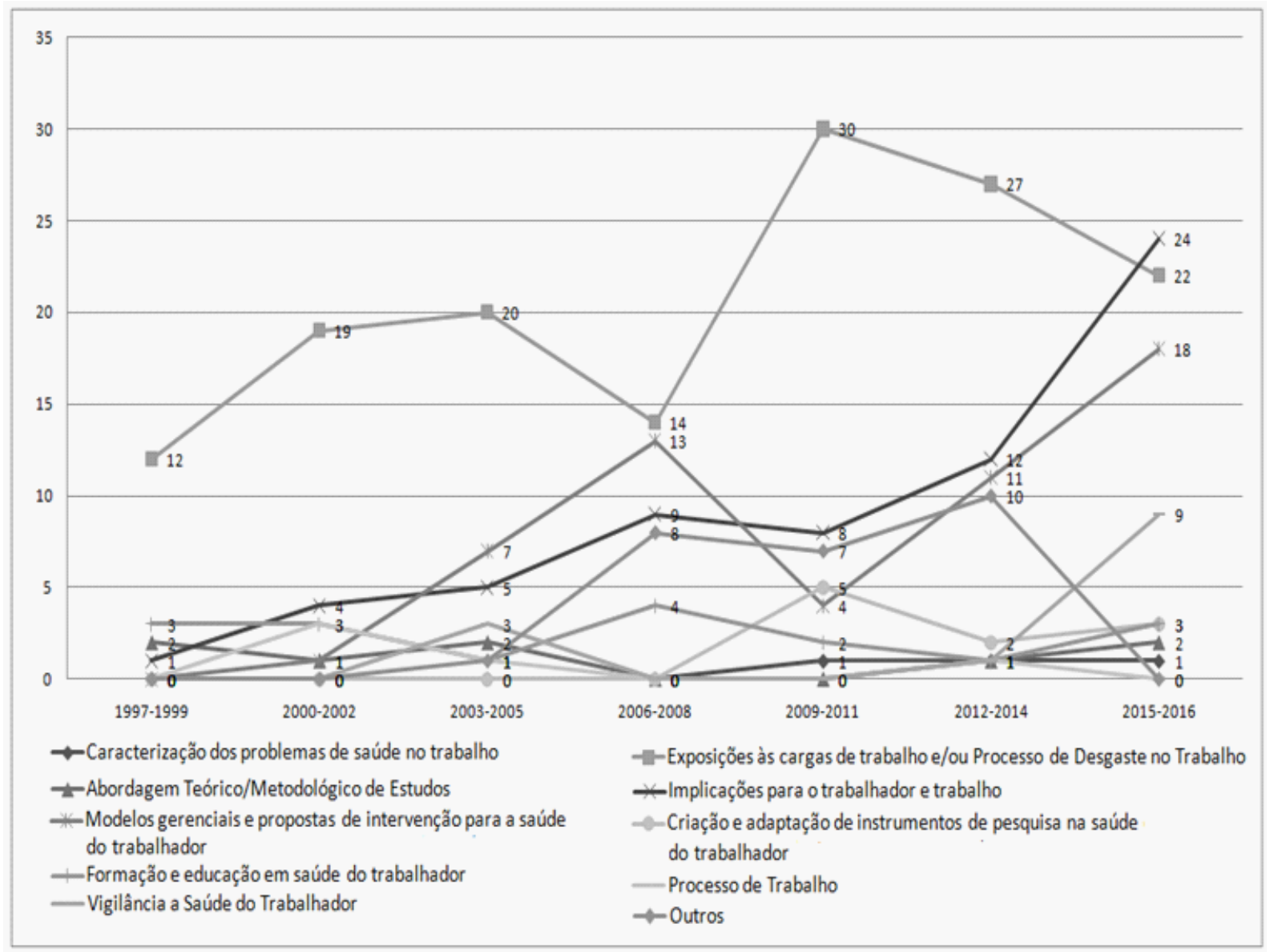

Figura 4 - Temas das publicações resultantes das atividades científicas do GESTES, entre 1997 e 2016, segundo o período de três anos. São Paulo, SP, Brasil, 2017

Observa-se que a temática "Exposições a cargas de trabalho e/ou Processo de desgaste no Trabalho" foi a mais predominante, seguida de uma variação das demais temáticas. Entretanto, nos últimos anos avaliados, destacam-se as temáticas "Implicações para o trabalhador e para o trabalho", "Modelos gerenciais e propostas de intervenção para a saúde do trabalhador" e "Vigilância a Saúde do Trabalhador".

A internacionalização das produções científicas do grupo é evidenciada na Figura 5, onde é mostrada a distribuição das publicações por países. 


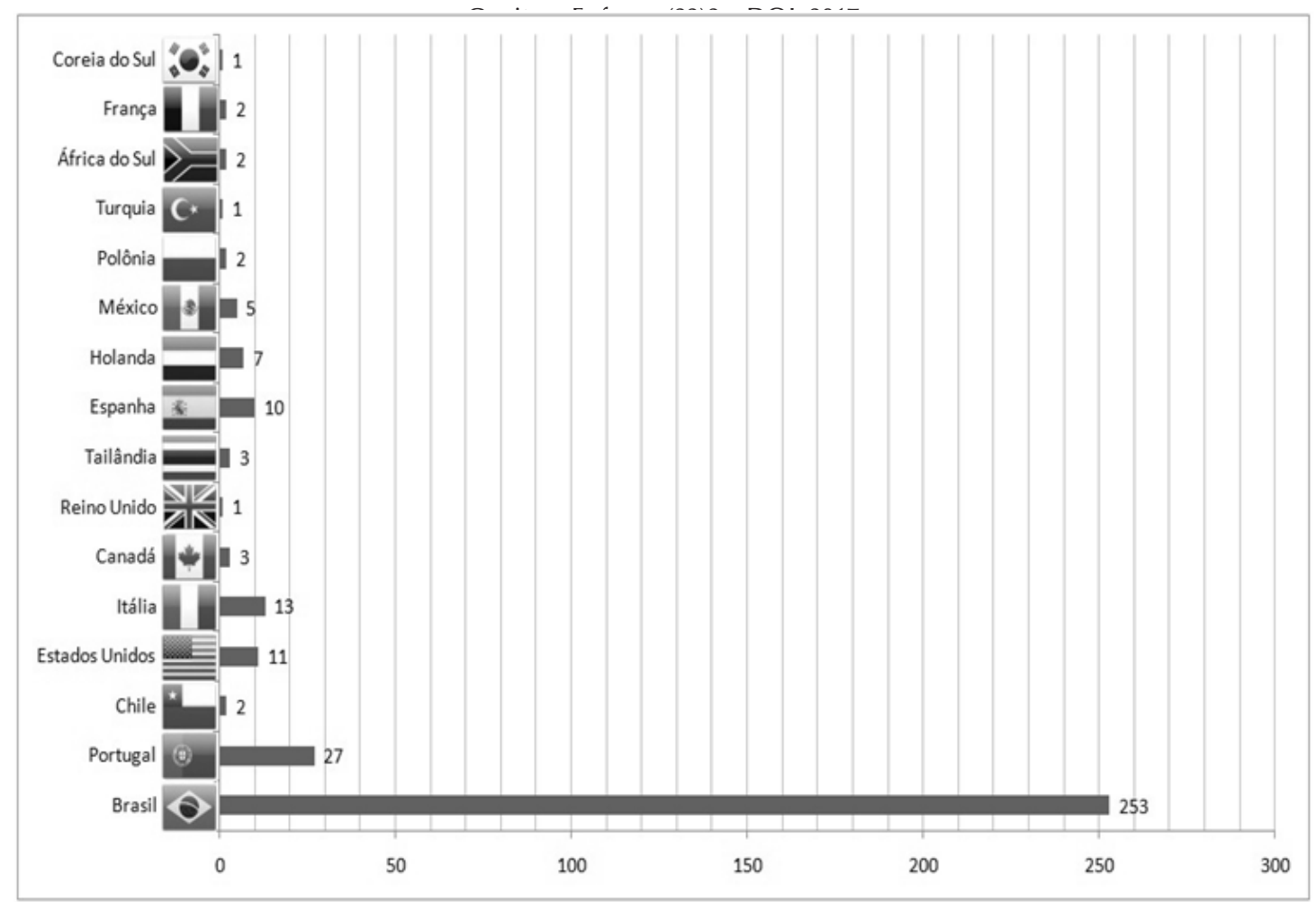

Figura 5 - Distribuição das produções científicas do GESTES por países, entre 1997 e 2016. São Paulo, SP, Brasil, 2017

O país com a maior quantidade de publicações é o Brasil (253), seguido de Portugal (27), Itália (13), Estados Unidos (11) e Espanha (10). É possível evidenciar a internacionalização dada a presença de publicações em vários países, em quase todos os continentes (África, América, Ásia e Europa).

\section{DISCUSSÃO}

O resgate e a análise da produção de conhecimento publicada sobre a saúde do trabalhador de enfermagem e de saúde gerada pelos integrantes do GESTES permitiu a identificação do perfil acadêmico de seus participantes, que inclui graduandos, pós-graduandos e pesquisadores, além da compreensão dos problemas e temáticas estudados.

O grupo de pesquisa está locado na EEUSP, Universidade Pública estadual, onde foi implantado o primeiro curso de graduação em enfermagem do estado de São Paulo, e que detém 51 grupos de pesquisa em enfermagem, sendo destaque no cenário nacional pela produção de conhecimento proveniente de cursos de graduação, pós-graduação e extensão ${ }^{(10)}$. Ressalta-se ainda o fato dos líderes integrarem a REPEGENF, o GESTES promove a articulação e a parceria como outros grupos e pesquisadores da temática, no cenário nacional ${ }^{(11)}$.

Considerando que o grupo acolhe alunos de graduação em enfermagem, promove a formação em pesquisa desses estudantes, assim o consumo dessa como importante instrumento para a prática de enfermagem. A pesquisa incrementa a prática baseada em evidências, a educação permanente e, consequentemente, a visibilidade da Enfermagem ${ }^{(12)}$. Essa valorização tem incentivado a participação dos alunos de graduação(13).

Desde a década de 1990, identifica-se o aumento dos cursos de doutorado nas Américas e Caribe, com maior esforço de internacionalização dos programas, um aumento no número de pesquisadores e de centros produtores de conhecimento de enfermagem ${ }^{(14)}$. A formação de doutores na enfermagem contribui significativamente para construção de um conhecimento social na área da saúde. Observase também um considerável crescimento do número de doutores na área, assim como a produção científica. O maior desafio ainda está na necessidade de as enfermeiras desenvolverem pesquisas experimentais que contenham propostas de mudanças para as práticas de saúde ${ }^{(15)}$. 
Ao observar a maior parte das publicações compostas por resumos e resumos expandidos, verificase a intencionalidade do grupo de divulgar os resultados de seus estudos em locais de disseminação científica coletiva, mantendo a valorização da publicação em periódicos científicos.

A produção científica crescente tem buscado e conquistado um significativo número de publicações internacionais, além das apresentações dos trabalhos científicos tanto por docentes, como discentes em eventos fora do país. A internacionalização é um caminho a ser trilhado pela enfermagem enquanto ciência. Porém, ao produzir resultados de pesquisa, o melhor meio de divulgação deve ser escolhido, a fim de suscitar novas pesquisas e informar aqueles envolvidos nos processos de tomada de decisões ${ }^{(16-17)}$.

O número de trabalhos científicos publicados na área de enfermagem tem aumentado consideravelmente. Atualmente, o aumento da produção do conhecimento é concomitante ao aumento da produção científica. Ao comparar a produção científica da área de Enfermagem entre os triênios de 2004 a 2006 e de 2007 a 2009, verificou-se um crescimento de 3.563 artigos, publicados em 373 periódicos, para 5.194 artigos publicados em 595 periódicos $^{(18)}$.

Com o desenvolvimento de estudos na temática abordada pelo grupo, resultando em diversas publicações, fica evidente a importância deste grupo para a enfermagem e para saúde do trabalhador, visto que a partir dessas evidências científicas é possível a viabilização de melhorias para o trabalho em enfermagem, assim como melhor alocação de recursos humanos e materiais no desenvolvimento das atividades laborais realizadas pela equipe de enfermagem.

O crescimento de publicações no grupo segue a tendência histórica de aumento de estudos na área de saúde do trabalhador, que iniciou nas décadas de 1970 e 1980, com foco em ergonomia, e ampliouse, posteriormente, com a incorporação de outras abordagens teórico-metodológicas, no final da década de 1980, com o uso da metodologia qualitativa, que passou a possibilitar análises e reflexões relacionadas à subjetividade e trabalho(3).

Em um levantamento das linhas de pesquisa dos grupos cadastrados no Diretório de Grupos de Pesquisa no Brasil Lattes, encontramos 15 que possuem a saúde do trabalhador como temática central. O fenômeno de crescimento de publicações na área pode ser observado na constituição destes grupos, onde se observa que $27 \%$ dos grupos de pesquisa na área possuem de 0 a 5 anos de atuação, $34 \%$ de 5 a 9 anos, $18 \%$ de 9 a 14 anos, $16 \%$ de 14 a 19 anos e $5 \%$ de 19 a 24 anos, com pico de aumento no período de 2006 a 2011 que coincide com a alimentação online da base de dados sobre os grupos de pesquisa no país.

Considerando-se a temática "Exposições a cargas de trabalho e/ou Processo de desgaste no Trabalho", estudos evidenciam as questões de carga de trabalho em enfermagem, relacionando-as ao desequilíbrio entre, de um lado, os recursos e competências e, de outro, as tarefas e expectativas, com a necessidade evidente de atuação dos gestores, em ações de alocação de recursos e atividades de educação ${ }^{(19)}$. Além da sobrecarga de trabalho já ter sido apresentada como relacionada diretamente com a segurança do paciente, com a ocorrência de eventos adversos a esta relacionados, há também relações bem estabelecidas com a saúde do trabalhador em enfermagem, sendo que a sobrecarga pode resultar em absenteísmo, rotatividade profissional e doenças ocupacionais ${ }^{(20-21)}$.

O trabalho, que é considerado elemento que compõe a identidade de um indivíduo, gerando significados presentes na relação com sua organização, e compondo vivências de prazer e sofrimento, é elemento determinante com implicações na saúde mental dos trabalhadores ${ }^{(22)}$. Estes são alguns dos importantes elementos que justificam a relevância da temática "Implicações para o trabalhador e para o trabalho".

A relevância das temáticas "Modelos gerenciais e propostas de intervenção para a saúde do trabalhador" e "Processo de trabalho" é evidenciada por estudos que reforçam a necessidade da verificação das lacunas entre o trabalho prescrito e o realizado, de reflexões sobre a relação entre o adoecimento e os níveis de demanda psicológica e de controle do trabalhador sobre o trabalho, da gestão participativa com a criação de espaços de discussão sobre as condições de trabalho, e do modelo de gestão do trabalho segundo lógica não maquínica ou burocrática ${ }^{(23-26)}$.

Ainda em relação à pesquisa em enfermagem, pesquisadores têm se debruçado na reflexão sobre 
o que e porque pesquisar, sendo foco de discussão no contexto nacional os Seminários Nacionais de Pesquisa em Enfermagem (SENPE) ${ }^{(27)}$. Este evento vem sendo realizado desde 1979, capitaneado pela Associação Brasileira de Enfermagem (ABEN) que tem como desafio, entre outros, colaborar com o processo de construção do conhecimento por meio da organização social da classe, por meio de profícuos debates acerca de seu devir. A Política Nacional de Ciência, Tecnologia e Inovação em Saúde (PNCTIS) e a Agenda Nacional de Prioridades de Pesquisa em Saúde (ANPPS) são também apontados como mecanismos para aumentar a seletividade, indução e fomento à pesquisa no país, respeitando as características regionais ${ }^{(28)}$. Ambos resultaram na indicação de 11 temas diretamente ligados à enfermagem, sendo eles:

\begin{abstract}
“... Saúde, Ambiente, Trabalho e Biossegurança em Enfermagem; Avaliação de Tecnologias de Enfermagem e Economia da Saúde; Investigação Clínica em Enfermagem; Gestão do Trabalho e Educação em Saúde; Sistemas e Políticas de Saúde; Cuidado de Enfermagem à Saúde do Adulto e do Idoso; Cuidado de Enfermagem à Saúde da Mulher; Cuidado de Enfermagem à Saúde da Criança e do Adolescente; Cuidado de Enfermagem à Saúde Mental; Cuidado de Enfermagem às Doenças Transmissíveis; Cuidado de Enfermagem às Doenças Não transmissíveis"(28).
\end{abstract}

Tais prioridades são possibilidades para nortear a criação de linhas de pesquisa, considerando-se o escopo da enfermagem, seu cenário nacional e seus paradigmas enquanto ciência, para bem delimitar seu campo de saber.

Ressalta-se ainda que as produções do GESTES tem seguido, ao longo desses 20 anos, perfil semelhante ao discutido no $18^{\circ}$ SENPE de 2015, que trouxe, em suas discussões, recomendações para que as pesquisas em enfermagem sejam mais próximas dos determinantes sociais do processo saúdedoença, assim como da investigação epidemiológica e de necessidades de saúde das populações ${ }^{(29)}$.

\title{
- CONCLUSÃo
}

Após 20 anos de existência e trabalho desenvolvido pelos seus membros, o Grupo de Estudos Sobre a Saúde do Trabalhador de Enfermagem e Saúde tem apresentado produção científica significativa, possibilitando publicações científicas e participação dos alunos e pesquisadores participantes em eventos nacionais e internacionais, dando assim a oportunidade de divulgação do conhecimento gerado.

Destaca-se que esses resultados representam significativas contribuições para o campo da enfermagem, por meio de fortalecimento da categoria a partir de pesquisas científicas, produções e intervenções que propõem melhorias nas condições de trabalho e qualidade de vida dos trabalhadores de enfermagem.

A fim de construir-se uma linguagem única e possibilitar um intercâmbio de informações, sem ruídos e/ou disparidades, compreende-se que há um desafio lançado ao processo de organização dos grupos de pesquisa no Brasil, especialmente no que tange à construção de redes que possibilitem debater a criação de uma "trama" comum entre as linhas de pesquisa e incremente a troca de informações e complementaridade mútua.

\section{REFERÊNCIAS}

1. Erdmann AL, Lanzoni GMM. Características dos grupos de pesquisa da enfermagem brasileira certificados pelo CNPq d 2005 a 2007. Esc. Anna Nery. [Internet] 2008;12(2) [acesso em 18 abr 2015]. Disponível: http://dx.doi. org/10.1590/S1414-81452008000200018.

2. Collet N, Schneider JF, Corrêa AK. A pesquisa em enfermagem: avanços e desafios. Rev. bras. enferm. [Internet] 2000;53(1) [acesso em 18 abr 2015]. Disponível: http://dx.doi.org/10.1590/S0034-71672000000100009.

3. de Oliveira EB, Lisboa MTL. Análise da produção científica da vertente saúde do trabalhador de enfermagem: subjetividade e trabalho. Rev. enferm. UERJ. [Internet] 2004;12(1) [acesso em 18 abr 2015]. Disponível: http:// www.facenf.uerj.br/v12n1/v12n1a04.pdf. 
[acesso em 20 abr 2015]. Disponível: http://dx.doi.org/10.3928/21650799-20120316-52.

5. Krug SBF, Assunção AN, Weigelt LD, Sehnem L, Alves LMS, Faller LA. Construindo caminhos, relatando vivências: a trajetória do grupo de estudos de pesquisa em saúde. Texto Contexto Enferm. [Internet] 2011;20(4) [acesso em 21 abr 2015]. Disponível: http://dx.doi.org/10.1590/S0104-07072011000400023.

6. Erdmann AL, Marziale MHP, Padreira MLG, Lana FCF, Pagliuca LMF, Padilha MI, et al. Evaluation of scientific periodicals and the brazilian production of nursing articles. Rev. Latino-Am. Enfermagem. [Internet] 2009;17(3) [acesso em 21 abr 2015]. Disponível: http://dx.doi.org/10.1590/S0104-11692009000300019.

7. Severinsson E. Rights and responsibilities in research supervision. Nurs Health Sci. [Internet] 2015;17(2) [acesso em 21 abr 2015]. Disponível: http://dx.doi.org/10.1111/nhs.12160.

8. Grupo de Estudos Sobre a Saúde do Trabalhador de Enfermagem e saúde: Diretório de Grupos de Pesquisa no Brasil Lattes. Conselho Nacional de Desenvolvimento Científico e Tecnológico. [Internet] Consulta parametrizada [acesso em 18 abr 2015]. Disponível: http://dgp.cnpq.br/dgp/espelhogrupo/3578989058528902.

9. Duran ECM, Toledo VP. Análise da produção do conhecimento em processo de enfermagem: estudo exploratório-descritivo. Rev. Gaúcha Enferm. [Internet] 2011;32(2) [acesso em 22 abr 2015]. Disponível: http:// dx.doi.org/10.1590/S1983-14472011000200004.

10. Canever BP, do Prado ML, Backes VMS, Lino MM. Characterization of research groups in nursing education in the state of são paulo. Texto Contexto Enferm. [Internet] 2014;23(1) [acesso em 16 mar 2016]. Disponível: http:// dx.doi.org/10.1590/S0104-07072014000100003.

11. Rocha CMF, Cassiani SHB. Nursing networks: strategies to strengthen research and extension studies. Rev. Gaúcha Enferm. [Internet] 2015;36(2) [acesso em 16 mar 2016]. Disponível: http://dx.doi.org/10.1590/19831447.2015.02.56420.

12. Piexak DR, Barlem JGT, da Silveira RS, Fernandes GFM, Lunardi VL, Backes DS. A percepção de estudantes da primeira série de um curso de graduação em enfermagem acerca da pesquisa. Esc. Anna Nery. [Internet] 2013;17(1) [acesso em 17 mar 2016]. Disponível: http://dx.doi.org/10.1590/S1414-81452013000100010.

13. Costa ACB, Chaves ECL, Terra FS, Monteiro LA. Perfil dos grupos de pesquisa de Enfermagem do Conselho Nacional de Desenvolvimento Científico e Tecnológico. Rev. Rene. [Internet] 2014;15(3) [acesso em 17 mar 2016]. Disponível: http://dx.doi.org/10.15253/2175-6783.2014000300012.

14. Cabral IG, Tyrrel MAR. Pesquisa em enfermagem nas Américas. Rev. bras. enferm. [Internet] 2010;63(1) [acesso em 20 mar 2016]. Disponível: http://dx.doi.org/10.1590/S0034-71672010000100017.

15. Rodrigues RAP, Erdmann AL, Silvia IA, Fernandes JD, Araújo TL, Vianna LAC, et al. Doctoral education in nursing in Brazil. Rev. Latino-Am. Enfermagem. [Internet] 2008;16(4) [acesso em 20 mar 2016]. Disponível: http:// dx.doi.org/10.1590/S0104-11692008000400003.

16. Costa IG, Parra MT, Hortense P, Opitz SP. Communication: a way to expand scientific nursing knowledge. In: Proceedings of the 8 Brazilian Nursing Communication Symposium [Internet] 2002 [acesso em 10 abr 2016]. Disponível: http://www.proceedings.scielo.br/scielo.php?script=sci_arttext\&pid=MSC000000005200200020005 7\&lng=en\&nrm=iso\&tlng=pt.

17. Figueiredo MLF. The internationalization of nursing research: a path to be followed. Rev. Enferm. UFPI. [Internet] 2013;2(3) [acesso em 10 abr 2016]. Disponível: http://www.ojs.ufpi.br/index.php/reufpi/article/view/1333.

18. Scochi CGS, Munari DB, Pedreira MLG, Padilha MI, Marziale MH. A importância da qualificação dos periódicos para o avanço da produção e visibilidade da pesquisa em enfermagem. Texto Contexto Enferm. [Internet] 2012;21(2) [acesso em 11 abr 2016]. Disponível: http://dx.doi.org/10.1590/S0104-07072012000200001.

19. Khademi M, Mohammadi E, Vanaki Z. Resources-tasks imbalance: Experiences of nurses from factors influencing workload to increase. Iran J Nurs Midwifery Res. [Internet] 2015;20(4) [acesso em 15 abr 2016]. Disponível: http://dx.doi.org/10.4103/1735-9066.160994.

20. de Magalhães AMM, Dall'Agnol CM, Marck PB. Nursing workload and patient safety - a mixed method study with an ecological restorative approach. Rev. Latino-Am. Enfermagem. [Internet] 2013;21(n.esp) [acesso em 12 mai 2016]. Disponível: http://dx.doi.org/10.1590/S0104-11692013000700019. 
21. Kang JH, Kim CW, Lee SY. Nurse-Perceived Patient Adverse Events depend on Nursing Workload. Osong Public Health Res Perspect. [Internet] 2016;7(1) [acesso em 13 mai 2016]. Disponível: http://dx.doi.org/10.1016/j. phrp.2015.10.015.

22. Cremonese GR, Motta RF, Traesel ES. Implicações do trabalho na saúde mental dos Agentes Comunitários de Saúde. Cad. psicol. soc. trab. [Internet] 2013;16(2) [acesso em 13 mai 2016]. Disponível: http://pepsic.bvsalud.org/ scielo.php?script=sci_arttext\&pid=S1516-37172013000200010.

23. Santana LL, Sarquis LMM, Miranda FMA, Kalinke LP, Felli VEA, Miniel VA. Health indicators of workers of the hospital area. Rev. bras. enferm. [Internet] 2016;69(1) [acesso em 07 jun 2016]. Disponível: http://dx.doi. org/10.1590/0034-7167.2016690104i.

24. Campos GWS. Cogestão e neoartesanato: elementos conceituais para repensar o trabalho em saúde combinando responsabilidade e autonomia. Ciênc. saúde coletiva. [Internet] 2010;15(5) [acesso em 12 mai 2016]. Disponível: http://dx.doi.org/10.1590/S1413-81232010000500009.

25. Trindade LL, de Pires DEP. Implications of primary health care models in workloads of health professionals. Texto Context Enferm. [Internet] 2013;22(1) [acesso em 12 mai 2016] Disponível: http://dx.doi.org/10.1590/S010407072013000100005.

26. Geco PBT, Magnago TSBS, Prochnow A, Beck CLC, Tavares JP. Utilização do modelo demanda-controle de Karasek na América Latina: uma pesquisa bibliográfica. Rev. enferm. UFSM. [Internet] 2011;1(2) [acesso em 12 mai 2016] Disponível: http://dx.doi.org/10.5902/217976922566.

27. da Fonseca RMGS, Oliveira RNG. Discussões sobre linhas de pesquisa nos Seminários Nacionais de Pesquisa em Enfermagem, 1979-2011. Rev. bras. enferm. [Internet] 2013;66(n.esp) [acesso em 15 fev 2016] Disponível: http:// dx.doi.org/10.1590/S0034-71672013000700009.

28. de Oliveira DC. Prioridades de pesquisa em enfermagem e as linhas de pesquisa: dando continuidade ao debate. Rev. enferm. UERJ. [Internet] 2014;22(5) [acesso em 07 fev 2016] Disponível: http://dx.doi.org/10.12957/ reuerj.2014.12771.

29. Vale EG, da Silva MJ. Research in nursing and new pathways based on SENPE. Rev. Bras. Enferm. [Internet] 2015;68(4) [acesso em 12 mai 2016] Disponível: http://dx.doi.org/10.1590/0034-7167.2015680401. 